measured ISGs). Score B consistently demonstrated stronger clinical associations in studies of diagnosis, prediction of progression to SLE in At-Risk individuals, response to rituximab and imaging-proven synovitis. Previous literature suggested that subsets of ISGs were explained by different interferon subtypes.

Objectives: To understand the determinants of IFN Score A and IFN Score B.

Methods: PBMCs and whole blood Tempus from 4 different healthy doners were stimulated with various cytokines and IFNs at 6- and 24-hour time points. RNA from both sample types were extracted and the expression of 26 interferon stimulated genes were measured using TaqMan and normalised to house-keeping gene PPIA. IFN Scores A and B were calculated as previously described [1]. To compare relative increase in expression with each condition, we calculated delta $\mathrm{Ct}$ fold change relative to non-stimulated. To represent greater expression as numerically higher positive values, relative expression was calculated as (fold change* ${ }^{*} 100$ ) +100 . Independent T-test calculated the significant differences between each condition compared to IFN $\alpha$ stimulation

Results: Table 1 shows differences between each condition compared with IFN $\alpha$ at the 6-hour time point. In both sample types, all conditions excluding IFN $\beta$ stimulation were shown to induce significantly lower expression of both scores compared to IFN $\alpha(p<0.01)$. At 6 and 24 hours, IFN $\alpha$ and IFN $\beta$ showed a strong induction of IFN score $A$ and $B$. At 6 hours, IFN $\gamma, \kappa$, and $\lambda$ induced IFN score $A$ and $B$ but weaker than IFN $\alpha$ and $\beta$ which was shown in both sample types. We did not observe a difference between the expression of these two scores according to interferon subtype. Other cytokines (TNF, IL-1 and IL-6) weakly induced expression of Score A and B. For IL-10 there was a possible discordant effect with increase in expression of Score B, but decreased expression of Score $A$, however these changes were small in magnitude.

Table 1.

\begin{tabular}{|c|c|c|c|c|}
\hline \multirow[t]{2}{*}{ Condition } & \multicolumn{2}{|l|}{ IFN Score A } & \multicolumn{2}{|l|}{ IFN Score B } \\
\hline & $\begin{array}{c}\text { Mean relative expression vs. } \\
\text { non-stimulated }\end{array}$ & $\begin{array}{c}\text { P value } \\
\text { (vs IFN } \alpha \text { ) }\end{array}$ & $\begin{array}{c}\text { Mean relative expression vs. } \\
\text { non-stimulated }\end{array}$ & $\begin{array}{c}\text { P value } \\
\text { (vs IFN } \\
\alpha)\end{array}$ \\
\hline \multicolumn{5}{|l|}{ PBMC } \\
\hline IFN $\alpha$ & 103.4 & - & 71.8 & - \\
\hline IFN $\beta$ & 97.1 & 0.373 & 64.7 & 0.352 \\
\hline IFN $\gamma$ & 37.5 & $<0.001$ & 29.5 & 0.002 \\
\hline IFN $\kappa$ & 16.6 & $<0.001$ & 3.1 & $<0.001$ \\
\hline IFN $\lambda$ & 24.8 & $<0.001$ & 11.1 & $<0.001$ \\
\hline IL-1 & 9.7 & $<0.001$ & 4.4 & $<0.001$ \\
\hline IL-6 & 4.5 & $<0.001$ & 4.1 & $<0.001$ \\
\hline IL-10 & -15.2 & $<0.001$ & 8.0 & $<0.001$ \\
\hline $\operatorname{TNF} \alpha$ & 9.6 & $<0.001$ & 1.2 & $<0.001$ \\
\hline \multicolumn{5}{|c|}{ Whole Blood } \\
\hline IFN $\alpha$ & 116.7 & - & 74.1 & - \\
\hline IFN $\beta$ & 116.6 & 0.997 & 73.2 & 0.894 \\
\hline IFN $\kappa$ & 23.0 & 0.001 & 7.9 & 0.003 \\
\hline IFN $\lambda$ & 23.0 & 0.001 & 7.6 & $<0.001$ \\
\hline
\end{tabular}

Conclusion: We found a difference in the induction of ISG expression between subtypes of Type I interferon, as well as other interferons and cytokines. However, the relative expression of IFN Score A and IFN Score B is not easily explained by the subtypes of interferon. We have also previously shown that these scores are both similarly expressed comparing different cell subsets. The explanation for the coordinated expression of these ISGs is therefore unclear and future work will explore the scores with a combination of conditions.

REFERENCES:

[1] El-Sherbiny, Y.M., et al. Scientific Reports, 2018. 8(1): p. 5793.

Disclosure of Interests: Zoe Wigston: None declared, Agata Burska: None declared, Miriam Wittmann Consultant of: Abbvie, Celgene, Janssen, L'Oreal, Novartis and Pfizer, Edward Vital Speakers bureau: Becton Dickinson and GSK, Consultant of: AstraZeneca, GSK, Roche/Genentech, and Sandoz, Grant/ research support from: AstraZeneca, Roche/Genentech

DOI: 10.1136/annrheumdis-2021-eular.1100

\section{POS0180 \\ TYPE I INTERFERON DEPENDENT HSA-MIR-145- 5P DOWNREGULATION MODULATES MUC1 AND TLR4 OVEREXPRESSION IN SALIVARY GLANDS FROM PRIMARY SJÖGREN'S SYNDROME PATIENTS}

D. Jara ${ }^{1}$, S. Aguilera $^{2}$, P. Carvajal ${ }^{1}$, I. Castro $^{3}$, M. J. Barrera ${ }^{4}$, S. González ${ }^{5}$, C. Molina ${ }^{4}$, M. J. González ${ }^{1}{ }^{1}$ Instituto de Ciencias Biomédicas, Facultad de Medicina, Universidad de Chile, Programa de Biología Celular y Molecular Santiago, Chile; ${ }^{2}$ Clínica INDISA, Rheumatology, Santiago, Chile; ${ }^{3}$ Facultad de Medicina, Universidad de Chile, Departamento de Tecnología Médica, Santiago, Chile; ${ }^{4}$ Universidad San Sebastián, Facultad de Odontología, Santiago, Chile; ${ }^{5}$ Facultad de Ciencias, Universidad Mayor, Escuela de Odontología, Santiago, Chile

Background: Primary Sjögren's syndrome (pSS) is an autoimmune and inflammatory disease that mainly affects the salivary glands (SG) and is characterized by an overactivation of the type I interferon pathway (IFNs I). IFNs I are known to regulate the levels of microRNAs (miRNAs), non-coding RNAs whose levels are altered in pSS. IFNs I can decrease the levels of miR-145-5p, a miRNA with anti-inflammatory roles that has been reported downregulated in SG of from pSS patients [1]. This miRNA has TLR4 and MUC1 transcripts as predicted targets. MUC1 and TLR4 are two proteins overexpressed in SG of pSS patients that contribute, through various mechanisms, to the inflammatory state and glandular dysfunction [2-3]. Thus, we propose that IFNs I may contribute to a self-perpetuating inflammation loop through a hsa-miR-145-5p dependent MUC1 and TLR4 overexpression in the SG of pSS patients.

Objectives: to evaluate whether mRNA levels of MUC1 and TLR4 are modulated by hsa-miR-145-5p in a IFNs I dependent manner.

Methods: 13 pSS patients and 9 controls SG biopsies were analyzed. hsa-miR145-5p levels were determined by TaqMan assays and MUC1, TLR4, IFN- $\alpha$ and IFN- $\beta$ mRNA levels by RT-qPCR. Additionally, in vitro assays using type IFNs and chemically synthesized hsa-miR-145-5p mimics and inhibitors were performed to study its effect on MUC1 and TLR4 expression. JAK1 and STAT1 mRNA levels were also measured.

Results: By Taqman assays we validated the decreased hsa-miR-145-5p levels $(p=0.0001)$ in SG of pSS patients compared to controls. The decreased hsa-miR-145-5p levels correlated inversely with the increased mRNA levels of IFN- $\beta(p=0.0192)$ in SG of pSS-patients. The hsa-miR-145-5p downregulation also correlated inversely with the overexpression of its predicted targets MUC1 $(p=0.010)$ and TLR4 $(p=0.0004)$. In vitro assays showed that IFN- $\beta$ induces the overexpression of JAK1 $(<p=0.0001)$ and STAT1 $(p=<0.0001)$ leading to the downregulation of hsa-mir-145-5p $(p=<0.0001)$ and increased MUC1 $(p=<0.0001)$ and TLR4 mRNA levels $(p=<0.0001)$. Functional assays suggest a regulation of hsa-miR-145-5p on MUC1 and TLR4 expression as MUC1 and TLR4 mRNA levels were decreased in HSG cells transfected with hsa-miR-513c-3p mimic and increased in HSG cells transfected with the miRNA inhibitor.

Conclusion: Our findings suggest that IFNs I could induce the downregulation of hsa-miR-145-5p leading to the overexpression of MUC1 and TLR4 in SG from pSS patients. TLR4 is activated by ectopic mucins in the SG extracellular matrix from pSS patients which induces pro-inflammatory cytokines secretion [3]. Furthermore, the high levels of the MUC1-SEC and MUC1-Y isoforms observed in SG from pSS patients may favor cytokine synthesis through the immuno-enhancing peptide of MUC1-SEC or through the formation of a MUC1-SEC/MUC1-Y complex [4]. Therefore, IFNs I may contribute to the development of SS through amplification and perpetuation of inflammation due to a hsa-miR-145-5p dependent MUC1 and TLR4 overexpression.

\section{REFERENCES:}

[1] I. Alevizos, et al,. Arthritis Rheum, 2011;63:535-44.

[2] HH. Sung, et al,. Oral Dis. 2015;21(6):730-8.

[3] MJ. Barrera, et al,. Rheumatology (Oxford). 2015;54(8):1518-27

[4] LM. Herbert, et al,. Cancer Res. 2004;64(21):8077-84.

Acknowledgements: Fondecyt 1210055, Fondecyt 1160015, Fondecyt Iniciación 11170049, Fondecyt Iniciación 11201058, CONICYT fellowship (DJ, PC) Disclosure of Interests: None declared

DOI: 10.1136/annrheumdis-2021-eular.2651 